\title{
INTERTEMPORAL ASSET PRICING WITHOUT CONSUMPTION DATA: EMPIRICAL TESTS
}

\author{
Yuming Li \\ The University of Michigan \\ and \\ California State University, Fullerton
}

\begin{abstract}
In this paper I conduct tests of an intertemporal asset pricing model using variables that forecast stock returns as the risk factors. I document that the forecasting variables are priced so that expected excess returns are related to their conditional covariances with the forecasting variables. The variability in the covariance risk fails to explain the cross-sectional and time-series variation in expected stock returns. Evidence rejects restrictions on the prices of covariance risk imposed by the model with constant volatilities. I also find that an extended model that allows time-varying conditional volatilities is misspecified.
\end{abstract}

\section{Introduction}

The intertemporal asset pricing model (I-CAPM) (Merton (1973), Cox, Ingersoll, and Ross (1985)) implies a relation between expected stock returns and sensitivities to risk factors. Empirical studies document that several economic variables are priced risk factors that explain cross-sectional differences in expected stock returns. Since recent research documents that stock returns are predictable, subsequent studies of the I-CAPM (e.g., Ferson and Harvey (1991)) relate time-varying expected returns and risk to variables that can forecast stock returns. These studies indicate that the risk premia for the economic variables vary with the forecasting variables, but they do not capture all of the predictable variation in expected stock returns.

Campbell (1993a) argues that the risk factors in the I-CAPM should be the forecasting variables instead of the economic variables used in the earlier literature. ${ }^{1}$ Using a representative agent model with the nonexpected utility function proposed by Epstein and Zin (1991) and a log-linear approximation to

The helpful comments of an anonymous referee and the editor Robert Hansen are appreciated.

${ }^{1} \mathrm{Li}$ (1992) argues the variables contained in the information set should include the state variables. 
the budget constraint, Campbell shows that expected asset returns are related to their conditional covariances with the forecasting variables. If the volatilities of asset returns and consumption are constant over time, the prices of covariance risk are functions of the coefficient of relative risk aversion, the ratio of consumption to wealth, and the coefficients of relative importance of each of the variables for forecasting future market returns. Otherwise, the risk prices are also related to a coefficient for the conditional volatilities of asset returns and consumption. Following the suggestion of Campbell (1993a), I propose and implement tests of the I-CAPM using the forecasting variables as the risk factors. ${ }^{2}$ These variables are a yield spread measuring term premium, a yield spread for default risk, the dividend yield, the one-month Treasury bill rate, and the lagged market return.

The estimation methodology in this paper is similar to Harvey's (1989) approach to testing the asset pricing model with time-varying conditional covariances. Harvey applies the approach to the market-based CAPM and concludes the time-varying expected stock returns captured by the forecasting variables are related to but are not explained by the conditional covariances with the market return.

The results here indicate that expected returns are significantly related to their conditional covariances with the forecasting variables. However, the evidence rejects the hypothesis that the forecasting variables are the risk factors that explain the cross-sectional and time-series variation in expected stock returns. Evidence also rejects restrictions on the prices of covariance risk imposed by Campbell's (1993a) model with constant volatilities. Although a specification test fails to reject restrictions on the risk prices imposed by an extension of the model that allows time-varying conditional volatilities, the negative sign on the estimated risk aversion coefficient indicates the extended model is also misspecified.

\section{Model}

In the I-CAPM of Merton (1973), Cox, Ingersoll, and Ross (1985), and Campbell (1993a), among others, expected asset returns are related to some risk factors or the state variables that follow a joint self-generating stochastic process. I use the vector autoregressive approach to describe the evolution of the state variables. I assume the real return on the market can be written as the first element of a $K \times 1$ state vector $\mathbf{y}_{i}$. The other elements include variables that are relevant for describing the state of the economy. The $K \times 1$ state vector $\mathbf{y}_{\text {t }}$ is assumed to follow a first-order vector autoregression (VAR):

${ }^{2}$ Campbell (1993b) uses the model to interpret the cross-sectional pattern of stock and bond returns. 


$$
\mathbf{y}_{t}=\xi+\Phi\left(\mathbf{y}_{t-1}-\xi\right)+v_{t},
$$

where $\xi$ is a $K \times 1$ vector of unconditional means, $\Phi$ is a $K \times K$ matrix of constant coefficients, and $v_{t}$ is a $K \times 1$ vector of residuals. I assume the residual vector satisfies $E\left[v_{T} \mid \mathbf{y}_{t-}\right]=0$ and $E\left[v_{t} v_{t-s}^{\prime}\right]=0$ for $s \neq 0$. Although a first-order VAR is assumed, a higher-order VAR can be rewritten as a first-order VAR. Hence, the assumption that the VAR is first-order is not restrictive.

Following Epstein and Zin (1991), the objective function for an infinitely lived representative agent is defined recursively,

$$
V_{t}=U\left[C_{t}, E_{t} V_{t+1}\right]=\left[(1-\beta) C_{t}^{1-\frac{1}{\sigma}}+\beta\left(E_{t} V_{t+1}\right)^{\frac{1-\frac{1}{\sigma}}{1-\gamma}}\right]^{\frac{1-\gamma}{1-\frac{1}{\sigma}}},
$$

where $E_{t}$ is the conditional expectation operator, $C_{t}$ is the total consumption in period $t, \gamma$ is the coefficient of relative risk aversion, $\sigma$ is the elasticity of intertemporal substitution, and $\beta$ is a discount factor. When $\gamma=1 / \sigma$, (2) specializes to the familiar expected utility specification:

$$
V_{t}=(1-\beta) E_{t}\left[\sum_{j=1}^{\infty} \beta^{j} C_{t+j}^{1-\gamma}\right]
$$

Assuming the ratio of consumption to wealth is constant over time, Campbell (1993a) shows that revisions in consumption are a linear function of revisions in current and future market returns. When the market return and other state variables are described by a VAR as in (1), Campbell shows that the expected return on asset $i$ in period $t, r_{i t}$, in excess of a one-period Treasury bill rate is a linear function of the conditional covariances of the asset return with the state vector: ${ }^{3}$

$$
E_{t-1} r_{i t}=\sum_{k=1}^{K} \operatorname{cov}_{t-1}\left(r_{i i}, v_{k t}\right) q_{k},
$$

where $\mathbf{q}=\left(q_{1}, \ldots, q_{K}\right)^{\prime}$ is a vector of prices of covariance risk. Although the asset pricing model implies asset returns are linear in conditional covariances, the risk prices in general are functions of the indirect utility function. In Campbell's

\footnotetext{
'In Campbell's (1993a) model, expected excess returns are also related to the variances of returns with continuous compounding. For returns with simple compounding, expected excess returns are only related to the covariances.
} 
(1993a) model, the risk prices are explicitly defined functions of the parameters of the state vector and other parameters in the investor's objective function.

Specifically, define $\lambda^{\prime}=\mathbf{e}_{1}^{\prime} \rho \Phi(\mathbf{I}-\rho \Phi)^{-1}$, a nonlinear function of the VAR coefficients, where $\mathbf{e}_{1}$ is a $K \times 1$ vector whose first element is one and whose other elements are zero, $\mathbf{I}$ is a $K \times K$ unit matrix, and $\rho$ is the constant ratio of reinvested wealth to wealth. Campbell (1993a) argues that the elements of the vector $\lambda$ measure the importance of each state variable in forecasting future market returns. Specifically, $\lambda_{k}$ is the discounted sum of revisions in expectations about market returns in each future period for an unexpected increase in the $k^{\text {th }}$ state variable. If a particular element of $\lambda_{k}$ is large and positive, a shock to the $k^{\text {th }}$ variable indicates an important piece of good news about future investment opportunities.

The risk prices are functions of the vector $\lambda$ and the parameters of the investor's objective function. When the joint distribution of asset returns and consumption is lognormal and homoskedastic, the risk prices are given by:

$$
\begin{aligned}
& q_{1}=\gamma+(\gamma-1) \lambda_{1}, \\
& q_{k}=(\gamma-1) \lambda_{k}, \quad k=2, \ldots, K .
\end{aligned}
$$

Thus, the risk price of the market portfolio is affected by the serial correlation properties of the market return. If the coefficient of relative risk aversion is greater than one, a positive value of $\lambda_{1}$ implies the risk price of the market portfolio is larger than the coefficient of relative risk aversion. The risk price of the $k^{\text {th }}$ state variable is positive if $\lambda_{k}$ is positive; that is, innovations in that variable are associated with good news about future investment opportunities, given that the coefficient of risk aversion is greater than one.

In (5), the risk prices are related to the coefficient of relative risk aversion, $\gamma$; the ratio of reinvested wealth to wealth, $\rho$; and the parameters of the state variables. This holds only if all variances and covariances of asset returns and consumption are assumed to be constant over time. When this assumption is relaxed, the risk prices also depend on a parameter because of the conditional heteroskedasticity in asset returns and consumption.

The Euler's equation for the investor's objective function implies that

$$
E_{t-1}\left[\log \left(C_{t} / C_{t-1}\right)\right]=\mu_{t-1}+\sigma E_{t-1} r_{m,},
$$

where $r_{m t}$ is the market return and $\mu_{t-1}$ is a function of the variances and covariances of the market return and consumption. When $\mu_{t-1}$ is assumed to be linearly related to the expected return on the market, that is, 


$$
\mu_{t-1}=\mu_{0}+\mu_{1} E_{t-1} r_{m}
$$

the risk prices are given by

$$
\begin{aligned}
& q_{1}=\gamma+(\gamma-1-\psi) \lambda_{1}, \\
& q_{k}=(\gamma-1-\psi) \lambda_{k}, \quad k=2, \ldots, K,
\end{aligned}
$$

where $\psi=\mu_{1}(\gamma-1) /(1-\sigma)$. When the variances and covariances of asset returns and consumption are assumed to be constant $-\mu_{1}=0$ - the risk prices given by (8) are the same as those given by (5). Thus, if $\mu_{1}=0$, conditional heteroskedasticity in asset returns and consumption has a nonneutral effect on risk prices unless $\gamma=1$ or $\sigma=1$.

\section{Estimation Method and Tests}

Specification Test of the VAR

To test the asset pricing model, a correctly specified VAR must be identified. I use Hansen's (1982) generalized method of moments (GMM) to provide estimates of the VAR parameters and to conduct specification tests.

As indicated earlier, a higher-order VAR can be stacked into a first-order form. To illustrate this, let $y_{11}$ and $y_{2 t}$ be two state variables. Assume that in addition to the first lags of the state variables, $y_{1, t-1}$ and $y_{2, t-1}$, the second lag of the second state variable, $y_{2, t-2}$, is also useful for describing changes in the conditional means of the state variables. I can write a first-order VAR for $\mathbf{y}_{\mathbf{t}}=\left(y_{11}, y_{2 t}, y_{2,-1-1}\right)^{\prime}$ that includes $y_{1, t-1}, y_{2, t-1}$, and $y_{2, t-2}$ as explanatory variables:

$$
\left[\begin{array}{c}
y_{11}-\xi_{1} \\
y_{2 t}-\xi_{2} \\
y_{2, t-1}-\xi_{2}
\end{array}\right]=\left[\begin{array}{lll}
\phi_{11} & \phi_{12} & \phi_{13} \\
\phi_{21} & \phi_{22} & \phi_{23} \\
0 & 1 & 0
\end{array}\right]\left[\begin{array}{c}
y_{1, t-1}-\xi_{1} \\
y_{2, t-1}-\xi_{2} \\
y_{2, t-2}-\xi_{2}
\end{array}\right]+\left[\begin{array}{l}
v_{1 \prime} \\
v_{2 t} \\
0
\end{array}\right] .
$$

By assumption, the VAR residual vector $v_{t}=\left(\mathbf{y}_{t}-\xi\right)-\Phi\left(\mathbf{y}_{t-1}-\xi\right)$ satisfies $E\left[\left.v_{t}\right|_{t-1}\right]$ where $\mathbf{I}_{t-1}$ is the information set available at time $t-1$. Define the following orthogonality conditions:

$$
\mathbf{f}_{t}^{0}=v_{t} \otimes \mathbf{z}_{t-1}^{0}
$$


where $\otimes$ denotes the Kronecker product or element-by-element multiplication and $\mathbf{z}_{t-1}^{0}$ is an instrument set that includes a constant contained in the information set $\mathbf{I}_{t-1}$. Then, the law of iterated expectations implies that $E\left[\mathbf{f}_{t}^{0}\right]=0$. Let $\mathbf{g}$ be the sample average of $\mathbf{f}_{i}^{0}$. The GMM estimates of parameters $(\xi, \Phi)$ are chosen to make the sample average $\mathrm{g}$ as close to zero as possible by minimizing the quadratic form: $J=\mathbf{g}^{\prime} \mathbf{W g}$, where $\mathbf{W}$ is a weighting matrix. Hansen (1982) outlines a form of the weighting matrix that guarantees the GMM estimates are consistent and asymptotically normal. Given the weighting matrix, the standard errors of the parameter estimates can be calculated using Hansen's method. The weighting matrix in the GMM estimation is iterated to achieve possibly superior finite sample properties suggested by Ferson and Forester (1994).

In (9), when the number of orthogonality conditions exceeds the number of parameters to be estimated, the minimized value of the quadratic form is distributed $\chi^{2}$ under the null hypothesis with the degree of freedom equal to the number of orthogonality conditions minus the number of parameters to be estimated. The $\chi^{2}$ statistic known as the test of overidentifying restrictions can be used to test the specification of the VAR.

\section{Testing the I-CAPM with Time-varying Conditional Covariances}

To simplify the notation, I define $\mathbf{r}_{\text {, }}$ as an $N \times 1$ vector of excess returns with the $i^{\text {th }}$ element $r_{i r}$. As in Harvey (1989), I rewrite the asset pricing model (4) as:

$$
E_{t-1} \mathbf{r}_{t}=\operatorname{cov}_{t-1}\left(\mathbf{r}_{t}, v_{t}\right) \mathbf{q}
$$

I also use the GMM to estimate and test the I-CAPM with time-varying conditional covariances given by (10). Since

$$
\operatorname{cov}_{t-1}\left(\mathbf{r}_{t}, v_{t}\right)=E_{t-1}\left(\mathbf{r}_{t} v_{t}^{\prime}\right)
$$

(10) can be rewritten as

$$
E_{t-1} \mathbf{r}_{t}=E_{t-1}\left(\mathbf{r}_{t} v_{t}^{\prime}\right) \mathbf{q}
$$

I define a vector of unexpected returns:

$$
\varepsilon_{1}=\mathbf{r}_{1}-r_{1} v_{1}^{\prime} q
$$


I have a set of orthogonality conditions:

$$
\mathbf{f}_{t}{ }^{1}=\varepsilon_{t} \otimes \mathbf{z}_{t-1},
$$

where $\mathbf{z}_{t-1}$ is a vector of a constant and the lags of the state vector $\mathbf{y}_{l}$. The law of iterated expectations implies that $E\left[\mathbf{f}_{t}^{\prime}\right]=0$.

To test the I-CAPM, I combine the orthogonality conditions implied by the VAR, $\mathbf{f}_{t}^{0}$, and by the I-CAPM, $\mathbf{f}_{\imath}^{1}$, into the following system:

$$
\mathbf{f}_{t}=\left[\begin{array}{c}
\mathbf{f}_{t}{ }^{0} \\
\mathbf{f}_{t}{ }_{t}
\end{array}\right]=\left[\begin{array}{c}
{\left[\mathbf{y}_{t}-\xi-\Phi\left(\mathbf{y}_{t-1}-\xi\right)\right] \otimes \mathbf{z}_{t-1}^{0}} \\
\left(\mathbf{r}_{t}-\mathbf{r}_{t} v_{t}^{\prime} \mathbf{q}\right) \otimes \mathbf{z}_{t-1}
\end{array}\right]
$$

The I-CAPM imposes some restrictions on the risk prices. Thus, the pricing model can be tested with or without imposing these restrictions. I consider three forms of the model. In the first form (model I), in addition to the parameters of the state vector, the risk prices are functions of the relative risk aversion coefficient and the consumption-wealth ratio, as given by (5). This model is implied by the assumption that the variances and covariances of consumption and returns are constant. In the second form (model II), the risk prices are also related to a coefficient due to conditional heteroskedasticity in asset returns and consumption, given by (8). In the third form (model III), the risk prices are assumed to be independent parameters. The estimation and tests of such a model are suggested by Harvey (1989). I first discuss the method of estimating and testing the third form of the model.

Given the orthogonality conditions, the parameter estimates of $\xi, \Phi$, and $\mathbf{q}$ can be obtained by minimizing the quadratic form $J=\mathbf{g}^{\prime} \mathbf{W g}$, where $\mathbf{g}$ is the sample average of orthogonality conditions, $\mathbf{f}_{t}$, and $\mathbf{W}$ is a weighting matrix. The minimized value of the quadratic form, distributed $\chi^{2}$ under the null, provides an overall goodness-of-fit test of the I-CAPM.

In (14), the instrument set $\mathbf{z}_{t-1}^{0}$ is chosen such that the total number of orthogonality conditions $\mathbf{f}_{t}^{0}$ is identical to the number of the VAR parameters. In this way, only the orthogonality conditions $\mathbf{f}_{\imath}^{1}$ contribute to the GMM overidentifying restrictions.

I estimate and test the first and second forms of the model in a similar way. For the first form, the risk prices are replaced by the right-hand sides of (5). For the second form, the risk prices are replaced by the right-hand sides of (8). I test the restrictions on the risk prices, (5) and (8), using the following likelihood ratio statistic (Newey and West (1987)): 


$$
\chi_{d}^{2}=\mathbf{g}_{r}{ }^{\prime} \mathbf{W}_{r} \mathbf{g}_{r}-\mathbf{g}_{u r}{ }^{\prime} \mathbf{W}_{r} \mathbf{g}_{u r}
$$

where $r$ represents restricted, $u r$ represents unrestricted, and $d$ is the degree of freedom equal to the number of parameter restrictions.

For example, to test the restrictions in (5) - that is, to test the restricted form (model I) against the unrestricted form (model III) - I first estimate the parameters of the two models and then compute the likelihood ratio statistic using a common weighting matrix calculated from the estimates of the parameters of the restricted model. In the restricted model, the risk prices $\mathbf{q}=\left(q_{1}, \ldots, q_{K}\right)^{\prime}$ are functions of the relative risk aversion parameter $\gamma$ and the consumption-to-wealth ratio $\rho$, in addition to the parameter of the state vector. Thus, the degree of the freedom of the $\chi^{2}$ statistic is $K-2$.

\section{Data and Summary Statistics}

\section{Data}

Following Harvey (1989), I study monthly returns on ten stock portfolios (Dec1-Dec10). All stock returns are provided by the Center for Research in Security Prices (CRSP) at the University of Chicago. The stock portfolios consist of deciles of stocks traded on the New York Stock Exchange (NYSE), ranked by the market value of equity outstanding at the beginning of each year. These portfolios are value weighted. Simple compound returns are used.

The risk factors are the market return and the variables that can forecast stock returns. The market return is the real return on the value-weighted portfolio of all NYSE stocks (RVW). ${ }^{4}$ Previous research (Fama and French (1989)) documents that a term premium variable, a measure of default risk, a dividend yield measure, and the one-month Treasury bill rate can predict stock returns. Harvey (1989) and Ferson and Harvey (1991), among others, use these to estimate time-varying expected returns and time-varying risk premia. The term premium (HB3) is the return for holding a ninety-day bill for one month less the onemonth Treasury bill rate. The measure of default risk (DEF) is the yield on Moody's Baa-rated bonds less the yield on Moody's Aaa-rated bonds. The dividend yield (DIV) is the sum of previous year's dividends on the valueweighted portfolio of all NYSE stocks, divided by the value of the portfolio.

\footnotetext{
${ }^{4}$ Much of the previous empirical work uses the excess market return rather than the real return. To be more closely related to the I-CAPM, I use the real return. The excess return is a reasonable proxy for the real return if the real return on the one-month bill is constant through time.
} 
Although RVW, HB3, DEF, and DIV are all real variables, the one-month Treasury bill rate (TB1) is a nominal variable that contains information about the expected real return on the one-month bill as well as expected inflation. The data for RVW, HB3, and DIV are calculated from CRSP return data. The real return is calculated by subtracting the Consumer Price Index inflation rate available from the CRSP from the nominal return. The corporate bond yields are obtained from Ibbotson and Associates for the period before the end of 1987 and from the Federal Reserve Board thereafter. The data set contains 384 monthly observations from January 1959 to December 1990.

\section{Summary Statistics and the Predictability of Returns}

Table 1 presents summary statistics for asset excess returns and risk factors. As shown in Panel A, the sample means and standard deviations of the size-based stock portfolios decrease from the portfolio with the smallest firms to the portfolio with the largest firms. Most of the stock portfolio returns have large first-order autocorrelations. Panel B gives summary statistics for the real return on the value-weighted market portfolio and other factors. The autocorrelations of DEF, DIV, and TB1 are larger and decline more slowly than the other variables. Panel $C$ reports cross-correlations among the factors. The correlation between DIV and TB1 is largest (0.72), followed by the correlation between DIV and DEF (0.56). The rest of the correlation estimates are much smaller.

To examine the predictive power of the factors, I regress excess returns on the first lags of the variables. Given the evidence on the January effect, asset excess returns are also regressed on a dummy variable for January (JAN) as an additional independent variable. The adjusted $R^{2}$ from the regressions are reported in the last column of Table $1 .^{5}$

The value-weighted market return has predictive power for returns on most of the stock portfolios. HB3, DIV, and TB1 can forecast stock returns. DEF, however, produces less significant coefficients. JAN produces significant coefficients for returns on small and medium firm stocks. Thus, JAN has predictive power for stock returns. This, however, does not imply JAN should be a state variable because it contains no unpredictable component and would not be a priced risk factor.

Without including JAN as one of the explanatory variables, the adjusted $R^{2}$ 's range from 8 percent to 12 percent for stock portfolios. When JAN is included, the adjusted $R^{2}$ is as high as 20 percent for the return on the smallest firm stock portfolio. These results suggest that significant proportions of monthly stock and bond returns are predictable. These results should not be surprising

${ }^{5}$ Since the regression results are similar to those reported in Harvey (1989), regression coefficients and standard errors are not reported. 
TABLE 1. Summary Statistics for Excess Returns" and the Factors, ${ }^{b} 1959-90$.

Autocorrelations at

\begin{tabular}{|c|c|c|c|c|c|c|c|c|c|c|}
\hline Variables & Mean $^{\mathrm{c}}$ & Std. Dev. ${ }^{\circ}$ & Lag 1 & Lag 2 & Lag 3 & Lag 4 & Lag 5 & Lag 12 & Lag 24 & Adj. $R^{2 \triangleleft}$ \\
\hline \multicolumn{11}{|c|}{ Panel A. Portfolios } \\
\hline Decl & 0.79 & 7.05 & 0.11 & -0.01 & -0.05 & -0.01 & 0.05 & 0.21 & 0.53 & 0.20 \\
\hline $\operatorname{Dec} 2$ & 0.72 & 6.32 & 0.13 & -0.02 & -0.04 & 0.01 & 0.04 & 0.17 & 0.04 & 0.19 \\
\hline Dec3 & 0.67 & 6.00 & 0.14 & -0.02 & -0.03 & -0.00 & 0.05 & 0.13 & 0.02 & 0.17 \\
\hline Dec4 & 0.66 & 5.73 & 0.16 & -0.04 & -0.03 & 0.01 & 0.04 & 0.10 & 0.02 & 0.16 \\
\hline $\operatorname{Dec} 5$ & 0.57 & 5.50 & 0.14 & -0.04 & -0.01 & -0.01 & 0.05 & 0.09 & 0.03 & 0.15 \\
\hline Dec6 & 0.58 & 5.30 & 0.15 & -0.04 & -0.03 & -0.00 & 0.05 & 0.05 & 0.00 & 0.14 \\
\hline $\operatorname{Dec} 7$ & 0.53 & 5.19 & 0.13 & -0.04 & -0.00 & -0.01 & 0.08 & 0.02 & -0.02 & 0.13 \\
\hline Dec8 & 0.56 & 5.01 & 0.10 & -0.06 & -0.03 & -0.02 & 0.08 & 0.03 & -0.01 & 0.12 \\
\hline Dec9 & 0.44 & 4.74 & 0.10 & -0.06 & -0.03 & 0.00 & 0.08 & 0.01 & -0.04 & 0.12 \\
\hline Declo & 0.33 & 4.23 & 0.02 & -0.04 & 0.01 & 0.03 & 0.11 & 0.04 & -0.01 & 0.08 \\
\hline
\end{tabular}

Panel B. Factors

\begin{tabular}{lrrrrrrrrr}
\hline RVW & 0.39 & 4.45 & 0.08 & -0.05 & 0.01 & 0.02 & 0.11 & 0.03 & -0.02 \\
HB3 & 0.07 & 0.11 & 0.29 & 0.04 & 0.04 & 0.03 & 0.07 & -0.04 & 0.03 \\
DEF & 0.09 & 0.06 & 0.86 & 0.78 & 0.72 & 0.66 & 0.64 & 0.37 & 0.18 \\
DIV & 0.31 & 0.07 & 0.97 & 0.95 & 0.92 & 0.90 & 0.87 & 0.71 & 0.60 \\
TBI & 0.49 & 0.23 & 0.96 & 0.92 & 0.88 & 0.86 & 0.84 & 0.72 & 0.50 \\
\hline
\end{tabular}

Panel C. Correlations

\begin{tabular}{lrlll}
\hline & RVW & HB3 & DEF & DIV \\
HB3 & -0.12 & & & \\
DEF & 0.00 & 0.34 & & \\
DIV & -0.10 & 0.30 & 0.56 & \\
TB1 & -0.11 & 0.23 & 0.42 & 0.72 \\
\hline
\end{tabular}

All returns are in excess of the one-month Treasury bill rate. Decl-Decl0 represent the decile of the smallestvalued firms to the decile of the largest-valued firms on the NYSE.

'The factors are: the real return on the value-weighted portfolio of all NYSE stocks (RVW), the return for holding a ninety-day bill for one month less the one-month Treasury bill rate (HB3), the yield on Moody's Baarated bonds less the yield on Moody's Aaa-rated bonds (DEF), the dividend yield on the value-weighted portfolio of all NYSE stocks (DIV), and the one-month Treasury bill rate (TB1).

'Means and standard deviations are in percentage per month.

${ }^{\mathrm{J}} \mathrm{Adj} . R^{2}$ are adjusted coefficients of determination for regressions of portfolio returns on the first lags of the factors and a January dummy variable.

given the substantial empirical evidence on the predictability of stock and bond returns.

Two other variables-the real return on the equally weighted stock portfolio (REW) and the inflation rate (INF)-are also possible factors. The predictive power of REW differs from that of RVW by less than 1 percent in terms of the $R^{2}$ for all of the portfolio returns. Without including TB1, I find INF 
can predict returns on all stock portfolios. When both TB 1 and INF are included, TB1 still has predictive power for stock returns but INF does not. To keep the total number of the state variables small, I do not add INF to the set of the state variables.

To examine the stability of the regression coefficients and the stability of the predictive power of the state variables, I divide the entire period from January 1959 to December 1990 into two subperiods with the breakpoint of August 1979. For the stock portfolios, the $p$-values for the Chow test of coefficient stability all exceed 12 percent. The adjusted $R^{2}$ 's are similar across the two subperiods. For example, when all of the state variables and JAN are included as explanatory variables, the adjusted $R^{2}$ is 21 percent for the smallest firm stock portfolio in the first subperiod and 20 percent in the second. These results are consistent with the findings of Harvey (1989), who examines returns from September 1941 to December 1987 with the same breakpoint. Thus the regression results suggest the state variables have significant predictive power for stock returns across the two subperiods. The empirical analysis below concentrates on the entire period. ${ }^{6}$

\section{Empirical Results}

\section{Estimation and Tests of the First-order VAR}

Table 2 provides estimates of coefficients in the first-order VAR model (1) for the risk factors RVW, HB3, DEF, DIV, and TB1. These estimates are obtained by setting the orthogonality conditions in (9) to zero. The instruments used to identify the parameters are the first lags of the risk factors plus a constant. In this way, the GMM system is exactly identified in that the number of orthogonality conditions equals the number of parameters to be estimated. The estimates of the unconditional means, $\xi_{j}$, of the risk factors are within 1 standard error from the sample means reported in Table 1. The estimates of the VAR coefficients, $\phi_{i j}$, suggest that most of the risk factors such as HB3, DIV, and TB1 are significant in predicting returns. These estimates also indicate the risk factors covary. For example, HB3 has forecasting power for RVW, DIV, and TB1.

I test the adequacy of the first-order VAR in modeling the stochastic process of the risk factors by including the first two or three lags of the risk factors as additional instruments. When the first-order VAR is misspecified, the residuals are likely to be correlated with these additional instruments. In such a case, the GMM test statistic is likely to be large. In Table 2, test 1 represents

\footnotetext{
"Harvey (1989) further evaluates the out-of-sample forecast power of similar variables for stock returns from September 1941 to December 1987. He finds that the out-of-sample $R^{2}$ 's are lower but not much lower than the in-sample $R^{2}$ 's.
} 


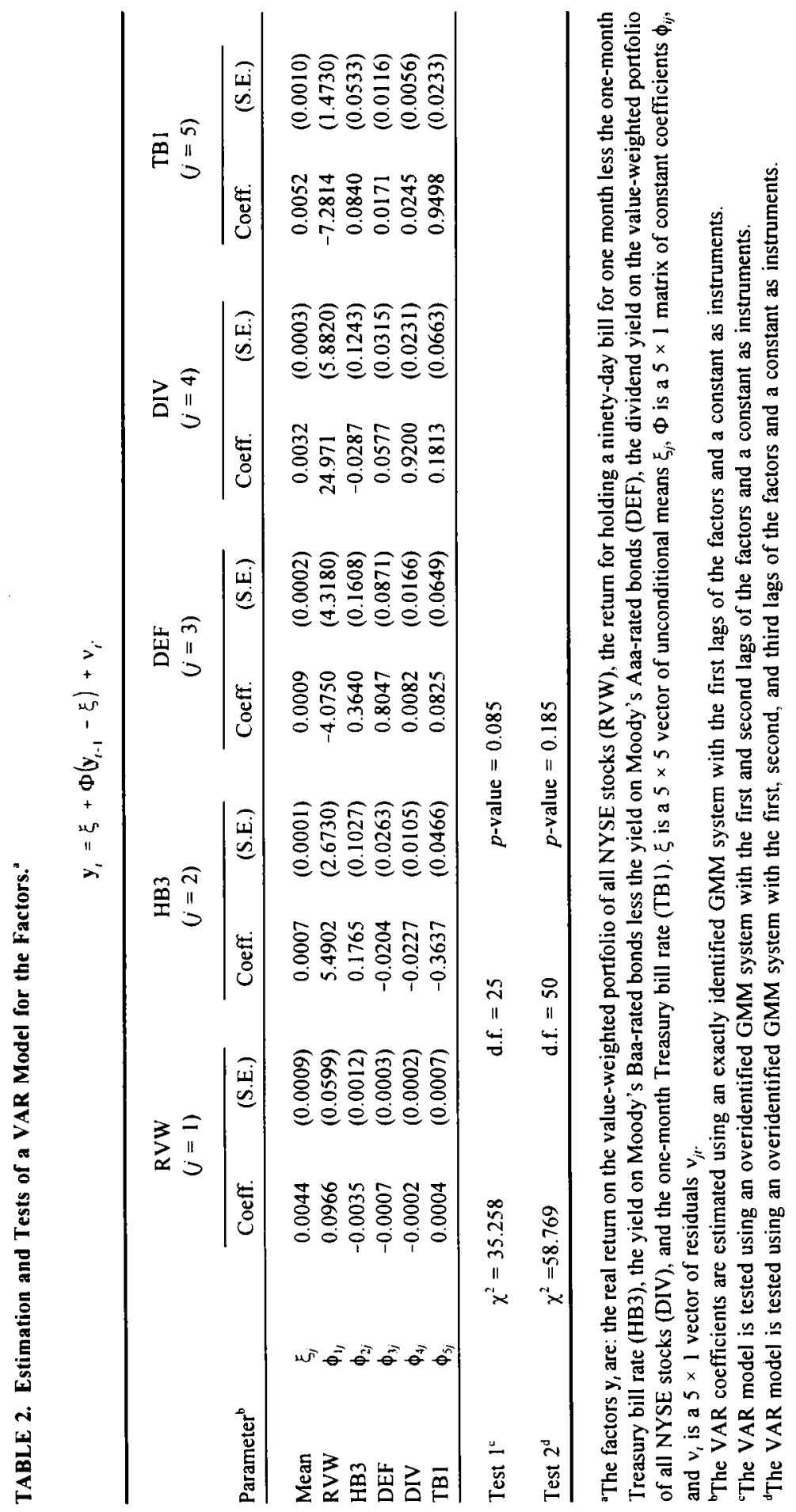


the test of GMM overidentifying restrictions when the first and second lags of the risk factors are used as the instruments, and test 2 represents the test when the first three lags of the risk factors are used. Results from tests 1 and 2 do not reject the hypothesis that the residuals are uncorrelated with the second and third lags of the risk factors at the 5 percent level. Thus, the second and third lags of the risk factors do not seem to be important in capturing the movements of the conditional means of the risk factors. Hence, the first-order VAR is used throughout the rest of the paper.

\section{Tests of the I-CAPM with Time-varying Conditional Covariances}

As described in the previous subsection, the parameters of risk factors in a first-order VAR can be exactly identified using the first lags of all of the risk factors plus a constant as the instrument set $\mathbf{z}_{t-1}^{0}$. The instrument set $\mathbf{z}_{t-1}$ used to form the orthogonally conditions implied by each of the three forms of the ICAPM consists of the first three lags of the risk factors plus a constant.

Table 3 summarizes the parameter estimates and tests of the three forms of the I-CAPM with or without restrictions on the risk prices. Panel A reports the GMM estimates of the parameters of models. The upper portion of Panel B reports the results of the GMM tests of overidentifying restrictions; the lower portion reports the likelihood ratio tests of restrictions on the risk prices imposed by the I-CAPM.

Recall that in the first form of the I-CAPM (model I), the joint distribution of asset returns and consumption is assumed to be lognormally distributed with constant variances and covarainces. The risk prices, $q_{k}$, in this model are functions of the relative risk aversion coefficient, $\gamma$, and the coefficient specified by (5), $\lambda_{k}$. The coefficient $\lambda_{k}$ is in turn a function of the constant ratio of invested wealth to wealth, $\rho$, and autoregressive coefficients of the VAR model for the risk factors. Thus, in model $\mathrm{I}$, in addition to the parameters of the risk factors, only two other parameters - i.e., $\gamma$ and $\rho$-are estimated. The estimate of $\gamma$ is 1.01 with a standard error of 0.03 . This appears reasonable. The estimate of $\rho$ is 1.12 . This point estimate, however, is not statistically different from zero or one, because the standard error is as large as 3.59 .

The goodness-of-fit test statistic, distributed $\chi^{2}$, is 216.23 . This test includes 190 orthogonality conditions and 32 parameters to be estimated, leaving 158 degrees of freedom. The $p$-value is thus 0.01 percent. Overall, this form of the I-CAPM is strongly rejected.

I now examine the results for model II. In this form of the I-CAPM the consumption and asset returns are still assumed to be jointly lognormal, but conditional variances and covariances are not necessarily constant over time. The risk prices are functions of not only $\gamma$ and $\rho$, but also $\psi$, a coefficient that measures the importance of conditional heteroskedasticity in addition to the parameters of the risk factors. 
TABLE 3. Tests of the I-CAPM with Time-varying Conditional Covariances.

Panel A. Parameter Estimates ${ }^{b}$

\begin{tabular}{|c|c|c|c|c|}
\hline Model & Variable & Parameter & Coefficient & Standard Error \\
\hline \multirow[t]{2}{*}{ I } & $\mathrm{RRA}^{\mathrm{c}}$ & $\lambda$ & 1.0103 & 0.027 \\
\hline & $1-C / W^{d}$ & $\rho$ & 1.1175 & 3.592 \\
\hline \multirow[t]{3}{*}{ II } & $\mathrm{RRA}^{\mathrm{c}}$ & $\lambda$ & -7.7422 & 3.161 \\
\hline & $1-C / W^{d}$ & $\rho$ & 0.9186 & 0.041 \\
\hline & $\mathrm{CH}^{\mathrm{c}}$ & $\psi$ & 38.742 & 19.70 \\
\hline \multirow[t]{5}{*}{ III } & RVW & $q_{1}$ & -9.4986 & 3.749 \\
\hline & HB3 & $q_{2}$ & 144.07 & 50.48 \\
\hline & DEF & $q_{3}$ & 1583.8 & 256.0 \\
\hline & DIV & $q_{4}$ & -3715.8 & 1059. \\
\hline & TB 1 & $q_{5}$ & 160.94 & 109.4 \\
\hline \multicolumn{5}{|c|}{ Panel B. Tests } \\
\hline Model & Test & d.f. & $x^{2}$ & $p$-value \\
\hline I & GMM test & 158 & 216.23 & 0.0014 \\
\hline II & GMM test & 157 & 200.95 & 0.0102 \\
\hline III & GMM test & 155 & 199.70 & 0.0090 \\
\hline I vs. II & Likehood ratio test & 1 & 7.4300 & 0.0064 \\
\hline I vs. III & Likelihood ratio test & 3 & 23.418 & 0.00003 \\
\hline II vs. III & Likelihood ratio test & 2 & 1.2468 & 0.2642 \\
\hline
\end{tabular}

Note: Model I imposes restrictions on risk prices under homoskedasticity. Model II imposes restrictions on risk prices under heteroskedasticity. Model III imposes no restrictions on the risk prices.

'The factors are: the real return on the value-weighted portfolio of all NYSE stocks (RVW), the return for holding a ninety-day bill for one month less the one-month Treasury bill rate (HB3), the yield on Moody's Baarated bonds less the yield on Moody's Aaa-rated bonds (DEF), the dividend yield on the value-weighted portfolio of all NYSE stocks (DIV), and the one-month Treasury bill rate (TB1)

"The estimation and tests use the first lags of the factors and a constant as the instruments.

'RRA $(\gamma)$ is a coefficient of relative risk aversion.

${ }^{\mathrm{d}} \mathrm{C} / \mathrm{W}$ is the constant ratio of consumption to wealth. $\rho=1-\mathrm{C} / \mathrm{W}$ is thus the ratio of invested wealth to total wealth.

${ }^{\mathrm{r}} \mathrm{CH}(\psi)$ is a coefficient resulting from the conditional heteroskedasticity of asset returns and consumptions.

In Table 3, for model II, the estiamte of $\gamma$ is a significant -7.74 with a standard error of 3.16. This appears to be evidence against model II. The estimate of $\rho$ is 0.92 with a standard error of 0.04 . This estimate is 2 standard errors from zero and one, as expected. The size of the estimate suggests that only a small portion of the value of the stock market portfolio is consumed, leaving the remaining wealth reinvested. The estimate of $\psi$ is 38.74 with a standard error of 19.70. This suggests that asset returns and consumption are conditionally heteroskedastic and that conditional heteroskedasticity may have a strong effect on risk prices. 
The goodness-of-fit statistic for this model is 200.95 with 157 degrees of freedom. Thus, the model is not rejected at the 1 percent level. Compared with model I, the form of the I-CAPM that allows conditional heteroskedasticity fits stock returns much better than the one that assumes homoskedasticity. Indeed, under the null hypothesis that the more restricted form (model I) is correct, the likelihood ratio test of the restriction on the coefficient of conditional heteroskedasticity, $H_{0}: \psi=0$, produces a $\chi^{2}$ statistic of 7.43 with 1 degree of freedom, resulting in a $p$-value of 0.64 percent. Thus, model I is rejected in favor of model II at any conventional significance level.

The third form of the I-CAPM (model III), which imposes no restrictions on the risk prices, is also estimated and tested. Different starting values are used to search for the estimates of the risk prices that provide the smallest value of the GMM goodness-of-fit statistic. The smallest value of this statistic is obtained when the initial estimates of the risk prices are set to those implied by the parameter estimates for model II. The estimates of risk prices are all significantly different from zero, implying that conditional covariances with each of the forecasting variables help explain variation in expected returns.

The goodness-of-fit statistic for model III is 199.70 , which is only slightly below that for model II, and the significance of this test is similar to that for model II. Allowing the risk prices to be free parameters does not improve the fit much. In other words, the restrictions imposed by the I-CAPM on risk prices may not violate the data. These restrictions are formally tested using the likelihood ratio statistic. Under the null hypothesis that model II is correct, the test statistic is 1.25 with 2 degrees of freedom, resulting in a $p$-value of 26.4 percent. This indicates that restrictions on risk prices are consistent with the observed returns.

In contrast, when the most restricted model (model I) is tested against the unrestricted model (model III), the likelihood ratio statistic is 23.42 with three degrees of freedom, producing a $p$-value of less than 0.01 percent. Thus, the restrictions on the risk prices imposed by the I-CAPM assuming homoskedasticity are strongly rejected. This test provides further evidence that conditional heteroskedasticity in asset returns and consumption plays a crucial role in explaining the behavior of stock returns.

Given that the I-CAPM allows for conditional heteroskedasticity in asset returns and consumption fits the data well, the way risk prices are determined in the model deserves more consideration. Table 4 reports the estimates of $\lambda_{k}$ implied by the parameter estimates for model II and the associated $t$-statistics calculated as the square root (plus a sign) of the Wald statistic for testing the parameter restriction that $H_{0}: \lambda_{k}=0$ for each $k$.

Recall that for a large and positive $\lambda_{k}$, an unexpected rise in the $i^{\text {th }}$ risk factor implies upward revisions in expectations about future market returns. From Table $4, \lambda_{1}$ and $\lambda_{4}$ are positive but the rest of the $\lambda_{k}$ 's are negative. All of the point estimates are statistically significant. Given that the first risk factor is the 
TABLE 4. Tests for the Predictive Importance of the Factors.

\begin{tabular}{lccc}
\hline Variable & Parameter & Coefficient $^{\mathrm{b}}$ & t-statistic $^{\mathrm{c}}$ \\
\hline RVW & $\lambda_{1}$ & 0.0528 & 2.476 \\
HB3 & $\lambda_{2}$ & -0.5087 & -3.105 \\
DEF & $\lambda_{3}$ & -34.701 & -2.632 \\
DIV & $\lambda_{4}$ & 82.593 & 2.378 \\
TB1 & $\lambda_{5}$ & -2.8113 & -1.794 \\
\hline
\end{tabular}

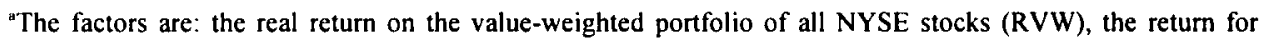
holding a ninety-day bill for one month less the one-month Treasury bill rate (HB3), the yield on Moody's Baarated bonds less the yield on Moody's Aaa-rated bonds (DEF), the dividend yield on the value-weighted portfolio of all NYSE stocks (DIV), and the one-month Treasury bill rate (TBI).

'The coefficients are implied by the parameter estimates for model II given in Table 3.

"The $t$-statistic is the square root of the Wald test statistic for the parameter restriction that $\lambda_{i}=0$ for each $l$.

market return, the positive value of $\lambda_{1}$ means that market returns are positively correlated. This is consistent with the positive autocorrelations of the market return at many of the lags, especially at lags 1 and 5 , as given in Table 1 . The positive value of $\lambda_{4}$ suggests that shocks to the dividend yield are good news about future investment opportunities. This is consistent with the view that dividend yield proxies for future expected returns (e.g., see Campbell and Shiller (1988) and Kothari and Shanken (1991)). The negative values of $\lambda_{2}, \lambda_{3}$, and $\lambda_{5}$ indicate that unexpected increases in the term spread, default spread, and shortterm Treasury bill rate are bad signals for future returns. This is also intuitively appealing.

\section{Conclusions}

In this paper I implement tests of the I-CAPM using variables that forecast returns as the risk factors. Tests reject the hypothesis that cross-sectional and time-series variation in expected returns can be explained by their conditional covariances with the forecasting variables. Evidence also rejects restrictions on the prices of covariance risk imposed by the I-CAPM of Campbell (1993a), in which expected returns are assumed to be constant over time. Although a specification test fails to reject restrictions on risk prices imposed by an extension of that model, which allows time-varying conditional volatilities, the negative sign of the estimated risk aversion coefficient indicates the extended model is also misspecified.

These results have implications for the correct specification of asset pricing models. Campbell's (1993a) model implies that the reward-to-risk parameters or the prices of covariance risk are constant through time. Harvey (1989) argues that the reward for market risk should vary through the business 
cycle. Bekaert and Harvey (1995) find countercyclical patterns in the reward for market risk. In light of the evidence in the literature, the rejection of the I-CAPM with constant risk prices in this paper may be attributed in part to the model's misspecification of risk prices.

The evidence here indicates that the forecasting variables are priced risk factors. This implies they may be used as substitutes for the economic state variables widely taken as the risk factors in tests of asset pricing models and applications of these models to the portfolio performance evaluation.

\section{References}

Bekaert, G. and C. R. Harvey, 1995, Time-varying world market integration, Journal of Finance 50, 403-44. Campbell, J. Y., 1993a, Intertemporal asset pricing without consumption data, American Economic Review 83, 487-512.

, 1993b, Understanding risk and return, Working paper, Princeton University and NBER.

Campbell, J. Y. and R. J. Shiller, 1988, The dividend price ratio and expectations of future dividends and discount factors, Review of Financial Studies 1, 195-228.

Cox, J. C., J. E. Ingersoll Jr., and S. A. Ross, 1985, An intertemporal general equilibrium model of asset prices, Econometrica 53, 363-84.

Epstein, L. and S. Zin, 1991, Substitution, risk aversion, and the temporal behavior of consumption and asset returns: An empirical analysis, Journal of Political Economy 99, 263-86.

Fama, E. F. and K. R. French, 1989, Business conditions and expected returns on stocks and bonds, Journal of Financial Economics 25, 23-49.

Ferson, W. E. and S. R. Forester, 1994, Finite sample properties of methods of moments in latent variable tests of asset pricing models, Journal of Financial Economics 36, 29-55.

Ferson, W. E. and C. R. Harvey, 1991, The variation of economic risk premia, Journal of Political Economy 99, 385-415.

Hansen, L. P., 1982, Large sample properties of generalized method of moments estimators, Econometrica 50, 1029-84.

Harvey, C. R., 1989, Time-varying conditional covariances in tests of asset pricing models, Journal of Financial Economics 24, 289-317.

Kothari, S. P. and J. Shanken, 1991, Stock retum variation and expected dividends: A time series and crosssectional analysis, Working paper, University of Rochester.

Li, Y., 1992, Expected stock returns, risk premia, and volatilities of economic factors, Unpublished Ph.D. dissertation, University of Chicago.

Merton, R., 1973, An intertemporal asset pricing model, Econometrica 41, 867-87.

Newey, W. K. and K. D. West, 1987, Hypothesis testing with efficient method of moments estimation, International Economic Review 28, 777-87. 